\title{
In-vitro bioaccessibility of antioxidant properties of bee pollen in Turkey
}

\author{
Dilek DULGER ALTINER ${ }^{1 *}$ (D), Sema SANDİKCİ ALTUNATMAZ² (D), Merve SABUNCU (D), Filiz AKSU² (D), \\ Yasemin SAHAN ${ }^{4}$ (D)
}

\begin{abstract}
The study aims to determine certain physicochemical properties (moisture, ash, titratable acidity, $\mathrm{pH}$, and color), total phenolic content, antioxidant capacity (CUPRAC, ABTS, DPPH) and bioaccessibility of 20 bee pollen samples (10 unpackaged and 10 trademarked products in their original packages) sold in Turkey. The total phenolic content of the bee pollen samples was detected as $147.10-462.02 \mathrm{mg} \mathrm{GAE} / \mathrm{g}$ and bioaccessibility ranged from $36.91 \%$ to $77.87 \%$. The highest total value for antioxidant capacity was assessed as 83.24-257.27 $\mu \mathrm{mol} \mathrm{TE} / \mathrm{g}$ by the CUPRAC method. When the bioaccessibility of the antioxidant capacity of bee pollen was compared among the methods, the highest value (95.76\%) was obtained for the sample $\mathrm{M}$ by the CUPRAC method. Antioxidant capacity and bioaccessibility of the unpackaged bee pollen samples were lower than those of the trademarked products. Based on overall evaluations, it can be deduced that bee pollen known for its potential beneficial effects on health may well be used as a functional food additive in the production of various food products with its high antioxidant and bioaccessibility features.
\end{abstract}

Keywords: bee pollen; antioxidant capacity; phenolics; bioaccessibility; functional food.

Practical Application: As a functional food component with high antioxidant properties and bioaccessibility, bee pollen can be used in the production of various foods and as a dietary supplement in nutrition.

\section{Introduction}

Pollen, which is a valuable food source for bees and bee larvae, is the product of the male reproductive cells of flowering plants. Flower pollen powder sticks to the whole body of worker bees while strolling (Borycka et al., 2015). Bee pollen is the mixture of flower pollen powder and the digestive enzymes of worker bees, which are collected on their legs and transported to the hive; therefore it contains flower grains, bee enzymes, and nectar sugars. Bee pollens are harvested through pollen traps placed in the entrance of the hives provided that the welfare of worker bees is maintained (Fadzilah et al., 2017; Alicic et al., 2014).

Bee pollen has drawn substantial attention in recent years with its pharmacological properties such as antimicrobial, antioxidant, antifungicidal, antiradiation, anticancer, and anti-inflammatory. Moreover, its preventive effects on arteriosclerosis, gastroenteritis, respiratory diseases, prostatic disorders, and allergic sensitization have been reported. Cardiovascular, gastrointestinal, immune system stimulating effects as well as antiaging properties of bee pollen have also been indicated. It is currently used in the treatment of sniffles, the flu, anemia, allergic diseases and ulcer and enteritis. It is called "the life-giving dust" owing to its extensive therapeutic effects and recognized as one of the functional foods in the food industry (Borycka et al., 2015; Carpes et al., 2007; Karadal et al., 2018; Kacániová et al., 2012; Guiné, 2015; Fadzilah et al., 2017; Karkar et al., 2018; Pascoal et al., 2014).
Certain factors such as unhealthy diet, environmental and industrial pollution and smoking trigger the production of free radicals in the cells and thus inhibit immune defense mechanisms and antioxidant enzymes. Oxidation induced in the body by free radicals produced during this process leads to oxidative stress, which causes lipid peroxidation, enzymatic deactivation, and accelerates the aging process. Demand for natural food products with high antioxidant content to alleviate metabolic problems has increased in recent years. Bee pollen, as well, is one of the high-quality food products with free-radical scavenging activity (Özkök \& Silici, 2017; Čeksteryté et al., 2016).

The key components with a potent antioxidant capacity that constitutes the beneficial medical properties of bee pollen are phenolic acids and flavonoids, which are plant-derived polyphenolic substances (Rzepecka-Stojko et al., 2015; Özkök $\&$ Silici, 2017). The mean percentage of phenolic content in bee pollen is $1.6 \%$. Phenolic compounds involve flavonoids, leukotriens, catechins and phenolic acids. Flavonoid content of bee pollen is $1.4 \%$ and consists primarily of kaempferol, quercetin, and isorhamnetin. Chlorogenic acid is the cardinal of phenolic acids and is found at a level of $0.2 \%$ (Komosinska-Vassev et al., 2015). The studies conducted on the antioxidant capacity of bee pollen pointed out a significant diversity in the antioxidant capacity of pollen grains, which is considered to be associated with the multiplicity of geographical area, environmental circumstances, 
and the plant species that the pollen grains were harvested from (Alicic et al., 2014).

Bee pollen exhibits such a biological diversity that it contains an approximate number of 200 different bioactive substances. The chemical composition of bee pollen includes proteins, amino acids, carbohydrates, lipids, fatty acids, phenolic compounds, enzymes, vitamins and minerals (Rzepecka-Stojko et al., 2015). Bee pollen contains 500-3000 mg mineral materials, 13-55 g carbohydrates, $10-40 \mathrm{~g}$ proteins, $1-10 \mathrm{~g}$ fat, $0.3-20 \mathrm{~g}$ dietary fiber, 20-100 $\mathrm{mg}$ vitamins and 40-3000 $\mathrm{mg}$ flavonoid glycosides per 100 gram of the sample (Bogdanov, 2006). Furthermore, it contains fat-soluble vitamins such as provitamin $A$, vitamin $E$, and vitamin $\mathrm{D}$ as well as water-soluble vitamins such as $\mathrm{B} 1, \mathrm{~B} 2$, and $\mathrm{C}$ at a level of $0.1 \%$ and $0.6 \%$, respectively. The amount of mineral in bee pollen is approximately $1.6 \%$ which includes both macro and microelements such as calcium, magnesium, sodium, phosphor and potassium, and iron, zinc, manganese, and selenium, respectively (Komosinska-Vassev et al., 2015; Sandıkçı Altunatmaz et al., 2017). It has been underlined in a study investigating the nutritional composition of bee pollen that it is a good nutritional source for the consumers particularly with numerous antioxidant vitamins and bioactive substances in its content (Sattler et al., 2015).

The research study was aimed to determine the amounts of total phenolic compounds, antioxidant capacities, bioaccessibilities and also certain physicochemical properties (moisture, ash, $\mathrm{pH}$, acidity, and color) of bee pollen used in Turkey as a food supplement.

\section{Materials and methods}

\subsection{Materials}

The study material of 20 bee pollen samples was collected from different sales points in Istanbul/Turkey. Ten of them were unpackaged products offered in plastic bags with a minimum of $100 \mathrm{~g}$ (Unpackaged (UPBp), A-J). The other ten samples were purchased in their original packages under different trademarks (Packaged (PBp), K-V). Trademarked products were all contained in properly sealed, dark-colored sun-proof packages. All samples were transferred to the laboratory in cold chain and kept at $+4{ }^{\circ} \mathrm{C}$ until the analyses.

\subsection{Methods}

All the reagents and chemicals used in the experiments were of analytical grade. The chemicals of antioxidant capacities and total phenolic contents were obtained from Sigma Chemical Co., Aldrich Chemicals Co., USA. All other chemicals used were obtained from the local suppliers. High-quality water obtained using a Milli-Q system (Millipore, Bedford, MA, USA), was used exclusively.

\section{Physico-chemical analysis of bee pollen}

Moisture (method no: 925.40), ash (method no: 950.49), acidity (Total) (method no: 935.57) and pH (Method No: 981.12) contents of the bee pollen samples were assessed according to the standard methods of Association of Official Analytical
Chemists (2000). The tests were performed at least in triplicate and mean values were reported.

The color measurement of bee pollen samples was carried out by Minolta Spectrophotometer CM-139 3600d (Osaka, Japan) based on CIE $\mathrm{L}^{*}, \mathrm{a}^{*}, \mathrm{~b}^{*}$ color system. The results were expressed using the CIELab system. The following parameters were determined: $L^{*}\left(L^{*}=0\right.$ black, $L^{*}=100$ white $), a^{*}$ - share of the green colour $\left(a^{*}<0\right)$ or red $\left(a^{*}>0\right), b^{*}$ - share of blue $\left(b^{*}<0\right)$ or yellow $\left(b^{*}>0\right)$. The measurement was repeated three times.

\section{Extraction of extractable, hydrolyzable, and bioaccessible} fractions

Three different extraction methods were applied for extractable, hydrolyzable and bioaccessible fractions. These methods were modified from those originally proposed by Vitali et al. (2009) and were used in the analyses of antioxidant capacity and total phenolic content. Briefly, $2 \mathrm{~g}$ were taken from each sample and these samples were extracted in $20 \mathrm{~mL}$ of $\mathrm{HCl}($ conc)/methanol/water $(1: 80: 10, \mathrm{v} / \mathrm{v})$ mixture at room temperature on an orbital shaker (JB50-D; Shanghai, China) (in $250 \mathrm{rpm}$ at $20^{\circ} \mathrm{C}$ ) for two hours. The obtained extracts were centrifuged (Sigma 3K 30, Germany) at $3500 \mathrm{rpm}$ for $10 \mathrm{~min}$. The supernatant was used as the extractable fraction in the analyses of total phenolic content and antioxidant capacity. The same procedure was repeated twice with the same solvent on the remaining part of the bee pollen samples. For hydrolyzable fraction, after extractable fraction, the residue was combined with $20 \mathrm{~mL}$ of methanol/ $\mathrm{H}_{2} \mathrm{SO}_{4}$ conc (10:1) and placed in a water bath at $85^{\circ} \mathrm{C}$ for $20 \mathrm{~h}$ before being cooled to room temperature. The mixtures were centrifuged at $3500 \mathrm{rpm}$ for $10 \mathrm{~min}$ at $4{ }^{\circ} \mathrm{C}$ in a centrifuge (Sigma 3K 30, Germany). The supernatants were collected and used as the hydrolyzable fraction for the analysis.

For the determination of bioaccessible fraction, investigated samples were processed by an in vitro digestive enzymatic extraction that mimics the conditions in the gastrointestinal tract according to the procedure of Vitali et al. 2009 with slight modifications. Briefly, $2 \mathrm{~g}$ of the samples were mix with $10 \mathrm{~mL}$ pure water and $0.5 \mathrm{~mL}$ pepsin and set to $\mathrm{pH} 2$ with $\mathrm{HCl}$ for the gastric environment and incubated in the shaking water bath at $37^{\circ} \mathrm{C}$ for $1 \mathrm{~h}$. Afterward, the $\mathrm{pH}$ level was increased to 7.2 to mimic the intestinal environment and $2.5 \mathrm{~mL} \mathrm{NaCl} / \mathrm{KCl}$ and $2.5 \mathrm{~mL}$ bile/pancreatin were added and then the samples were incubated in the shaking water bath at $37^{\circ} \mathrm{C}$ for $2.5 \mathrm{~h}$. Finally, the samples were centrifuged at $3500 \mathrm{rpm}$ for $10 \mathrm{~min}$ and supernatants were separated. The whole procedure was run in triplicate and all supernatants were stored at $20^{\circ} \mathrm{C}$ until used.

\section{Determination of Total Phenolic Contents (TPC)}

The extractable, hydrolyzable, and bioaccessible fraction of bee pollens were determined at $760 \mathrm{~nm}$ by using Shimadzu UV-1280 UV-VIS spectrophotometer according to the Folin-Ciocalteu method (Naczk \& Shahidi, 2004). Gallic acid was used as standard and the results were expressed as $\mathrm{mg} \mathrm{GAE} / \mathrm{g} \mathrm{dw}$. The total phenolic content was calculated as the sum of extractable and hydrolyzable fractions and bioaccessibility was calculated as the percentage of total phenolic content. The procedure was carried out three times for each extract. 


\section{Determination of Antioxidant Capacity (AC)}

Antioxidant capacities of extractable and hydrolyzable and bioaccessible fraction of the bee pollen samples were determined using radical cation decolorization assay (2,2'-azino-bis (3-ethylbenzothiazoline-6-sulphonic acid) (ABTS) (Apak et al., 2008), cupric ion reducing antioxidant capacity assay (CUPRAC) (Apak et al., 2004), free radical scavenging assay (2,2-diphenyl-1-picrylhydrazyl) (DPPH) (Brand-Williams et al., 1995), with slight modifications. All assays were repeated three times for each extract collected from the samples and absorbance of samples were measured by using spectrophotometer (Shimadzu UV-1280). A calibration curve was prepared, using Trolox (6-hydroxy-2,5,7,8-tetramethylchromane-2-carboxylic acid) and the results were expressed as $\mu \mathrm{mol} \mathrm{TE} / \mathrm{g} \mathrm{dw}$ for each method.

\subsection{Statistical analysis}

Data obtained from the analyses were statistically evaluated with a computer-based program JMP IN 7.0.0 (Statistical Discovery from SAS Institute Inc. (2007), the LSD (Least Significant Difference) test was performed to determine the significant difference between the mean values at the $\mathrm{p} \leq 0.05$ level.

\section{Results and discussion}

\subsection{Physicochemical properties}

The results of moisture, ash, $\mathrm{pH}$, titratable acidity and the color analyses of the bee pollen samples were shown in Table 1 . The mean moisture, ash, $\mathrm{pH}$, titratable acidity levels of the unpackaged (UPBp) products were determined as $20.98 \%$, $3.61 \%, 4.55$, and $3.67 \%$, while those of the originally packaged samples (PBp) were $21.36 \%, 3.89 \%, 4.80$, and 3.64\%, respectively (Table 1). No statistically significant difference was noted between the packaged and unpackaged bee pollen products in terms of physicochemical properties except for the titratable acidity $(\mathrm{p}<0.05)$. The ash contents of bee pollen samples found in this study were similar to those reported by Isık et al. (2019), which ranged from 2.14-2.18 g/100 g in hot air dried bee pollen samples.

Bárbara et al. (2015) reported similar results indicating that the mean moisture, ash, $\mathrm{pH}$, titratable acidity levels of 21 bee pollen (Melipona mandacaia) samples collected from two different regions of Bahia and Brazil were $36.0 \%, 4.9 \%, 3.49$, and $146 \mathrm{meq} \cdot \mathrm{kg}^{-1}$, respectively. Duarte et al. (2018) found similar $\mathrm{pH}$ values for bee pollen produced in the Alagoas, Northeast region of Brazil by M. subnitida and T. clavipes respectively 4.9 and 5.9.

Dried bee pollen moisture content was limited 6-10\% established by the local food standards by Argentina, Poland, Bulgaria, and Switzerland. However, in Brazil food standards moisture content was $30 \%$ in bee pollen and $4 \%$ in dehydrated pollen (Melo \& Almeida-Muradian, 2011). Almeida-Muradian et al. (2005) detected a mean level of $7.4 \%$ and $2.2 \%$ for moisture and ash, respectively in the study investigating the chemical composition of 10 different dried bee pollen products collected from the southern part of Brazil. According to the legal regulations in Brazil, the maximum permissible ash level in dried bee pollen is $4 \%$. (Brasil, 2001). In another study carried out in Brazil, bee pollen samples had 2.61-11.06\% moisture, 1.58-3.61\% ash contents and

Table 1. Physicochemical compositions of bee pollens.

\begin{tabular}{|c|c|c|c|c|c|c|c|}
\hline BPs & Moisture (\%) & Ash (\%) & $\mathrm{pH}$ & Acidity (\%) & $L^{*}$ & $a^{*}$ & $b^{*}$ \\
\hline $\mathbf{A}^{\star}$ & $20.74 \pm 0.06$ & $3.21 \pm 0.03$ & $4.84 \pm 0.05$ & $3.94 \pm 0.00$ & $57.91 \pm 0.97$ & $3.53 \pm 0.05$ & $23.43 \pm 0.71$ \\
\hline B & $20.62 \pm 0.00$ & $3.17 \pm 0.01$ & $4.35 \pm 0.04$ & $2.63 \pm 0.00$ & $57.94 \pm 0.53$ & $3.89 \pm 0.43$ & $24.55 \pm 0.65$ \\
\hline $\mathrm{C}$ & $20.65 \pm 0.42$ & $3.15 \pm 0.09$ & $4.71 \pm 0.03$ & $4.34 \pm 0.00$ & $60.01 \pm 0.55$ & $3.59 \pm 0.20$ & $25.94 \pm 0.43$ \\
\hline D & $21.64 \pm 0.00$ & $3.41 \pm 0.02$ & $4.44 \pm 0.06$ & $4.57 \pm 0.00$ & $59.84 \pm 0.48$ & $3.47 \pm 0.30$ & $25.69 \pm 0.55$ \\
\hline $\mathbf{E}$ & $20.80 \pm 0.41$ & $4.21 \pm 0.03$ & $4.37 \pm 0.08$ & $3.05 \pm 0.00$ & $57.77 \pm 0.53$ & $2.62 \pm 0.10$ & $22.23 \pm 0.45$ \\
\hline $\mathbf{F}$ & $19.27 \pm 0.39$ & $2.93 \pm 0.06$ & $4.82 \pm 0.03$ & $5.23 \pm 0.00$ & $55.75 \pm 0.84$ & $4.67 \pm 0.13$ & $24.70 \pm 0.56$ \\
\hline G & $22.40 \pm 0.07$ & $4.31 \pm 0.01$ & $4.55 \pm 0.04$ & $3.21 \pm 0.00$ & $52.21 \pm 1.25$ & $4.97 \pm 0.38$ & $22.57 \pm 0.89$ \\
\hline $\mathbf{H}$ & $22.35 \pm 0.15$ & $4.47 \pm 0.04$ & $4.30 \pm 0.04$ & $3.33 \pm 0.00$ & $47.73 \pm 0.47$ & $6.67 \pm 0.34$ & $19.92 \pm 0.30$ \\
\hline I & $20.94 \pm 0.39$ & $4.09 \pm 0.04$ & $4.57 \pm 0.03$ & $3.22 \pm 0.00$ & $57.48 \pm 1.16$ & $4.66 \pm 0.37$ & $23.73 \pm 0.46$ \\
\hline $\mathbf{J}$ & $19.93 \pm 0.06$ & $3.18 \pm 0.04$ & $4.55 \pm 0.04$ & $3.15 \pm 0.00$ & $57.56 \pm 0.74$ & $4.15 \pm 0.39$ & $26.64 \pm 0.40$ \\
\hline $\mathbf{K}^{* *}$ & $22.17 \pm 0.01$ & $3.80 \pm 0.01$ & $4.78 \pm 0.03$ & $2.51 \pm 0.00$ & $55.48 \pm 0.43$ & $3.73 \pm 0.45$ & $22.15 \pm 0.19$ \\
\hline $\mathbf{L}$ & $21.40 \pm 0.20$ & $3.52 \pm 0.03$ & $4.67 \pm 0.04$ & $3.25 \pm 0.00$ & $56.03 \pm 0.76$ & $2.89 \pm 0.13$ & $23.86 \pm 0.45$ \\
\hline $\mathbf{M}$ & $22.45 \pm 0.33$ & $4.14 \pm 0.01$ & $4.48 \pm 0.04$ & $5.82 \pm 0.00$ & $53.01 \pm 0.71$ & $5.08 \pm 0.05$ & $22.68 \pm 0.41$ \\
\hline $\mathbf{N}$ & $20.03 \pm 0.05$ & $3.67 \pm 0.01$ & $4.83 \pm 0.04$ & $5.34 \pm 0.00$ & $63.65 \pm 0.57$ & $1.54 \pm 0.24$ & $28.81 \pm 0.31$ \\
\hline $\mathbf{O}$ & $25.93 \pm 0.19$ & $4.87 \pm 0.01$ & $4.60 \pm 0.07$ & $3.26 \pm 0.00$ & $59.69 \pm 0.20$ & $4.50 \pm 0.12$ & $30.39 \pm 0.07$ \\
\hline $\mathbf{P}$ & $20.48 \pm 0.24$ & $5.03 \pm 0.01$ & $4.86 \pm 0.03$ & $4.65 \pm 0.00$ & $56.93 \pm 1.42$ & $3.80 \pm 0.18$ & $27.84 \pm 1.15$ \\
\hline $\mathbf{R}$ & $20.97 \pm 0.92$ & $3.82 \pm 0.06$ & $4.81 \pm 0.03$ & $2.51 \pm 0.00$ & $53.67 \pm 0.51$ & $6.30 \pm 0.31$ & $26.59 \pm 0.46$ \\
\hline $\mathbf{S}$ & $20.26 \pm 0.42$ & $3.37 \pm 0.04$ & $4.81 \pm 0.03$ & $2.51 \pm 0.00$ & $52.96 \pm 0.34$ & $5.47 \pm 0.16$ & $23.31 \pm 0.22$ \\
\hline $\mathbf{T}$ & $17.90 \pm 0.28$ & $2.92 \pm 0.01$ & $5.41 \pm 0.05$ & $3.99 \pm 0.00$ & $53.66 \pm 0.41$ & $7.19 \pm 0.39$ & $26.99 \pm 0.32$ \\
\hline $\mathbf{V}$ & $22.04 \pm 1.13$ & $3.73 \pm 0.06$ & $4.78 \pm 0.04$ & $2.61 \pm 0.00$ & $57.62 \pm 1.02$ & $3.40 \pm 0.08$ & $24.55 \pm 0.63$ \\
\hline Min-Max & $17.90-25.93$ & $2.92-5.03$ & $4.30-5.41$ & $2.51-5.82$ & $47.73-63.65$ & $1.54-7.19$ & $19.92-30.39$ \\
\hline \multicolumn{8}{|l|}{ Mean \pm SD } \\
\hline UPBp & $20.98 \pm 0.97^{\mathrm{a}}$ & $3.61 \pm 0.59^{\mathrm{a}}$ & $4.55 \pm 0.19^{\mathrm{a}}$ & $3.67 \pm 0.82^{b}$ & $56.42 \pm 3.76^{\mathrm{a}}$ & $4.22 \pm 1.11^{\mathrm{a}}$ & $23.94 \pm 2.02^{\mathrm{a}}$ \\
\hline PBp & $21.36 \pm 2.09^{\mathrm{a}}$ & $3.89 \pm 0.65^{\mathrm{a}}$ & $4.80 \pm 0.25^{\mathrm{a}}$ & $3.64 \pm 1.25^{\mathrm{a}}$ & $56.27 \pm 3.40^{\mathrm{a}}$ & $4.39 \pm 1.68^{\mathrm{a}}$ & $25.72 \pm 2.81^{\mathrm{a}}$ \\
\hline
\end{tabular}

Means with different superscripts in the columns indicate significant difference ( $<<0.05)$. Data are expressed as means \pm standard deviations (SD). Min: Minimum value of means, Max: Maximum values of means. BPs: Bee pollen samples. ${ }^{*} \mathrm{UPBp}$ : Unpackaged bee pollen samples A-J; ${ }^{* *} \mathrm{PBp}$ : Packaged bee pollen samples K-V. 
pH values of 4.60-5.90 (Marchini et al., 2006). Similar findings were reported in some other studies (Almeida-Muradian et al., 2005; Feás et al., 2012; Coronel et al., 2004; Vit \& Santiago, 2008). The chemical composition of bee pollen varies extensively depending on the plant species and growing conditions, agricultural practice, climate, botanical features and also harvesting and the storage conditions (Anjos et al., 2019a, b; Bonvehí et al., 2001; Almaraz-Abarca et al., 2004).

According to CIE color measurement system based on a three-coordinate diagram $\left(\mathrm{L}^{\star}, \mathrm{a}^{\star}, \mathrm{b}^{\star}\right)$ colors in numeric values located in different spaces of the diagram are evaluated as different colors as follows: $L^{*}=100$ white, $L^{*}=0$ black; positive $a^{*}$ red, negative $a^{\star}$ green; positive $b^{\star}$ yellow and negative $b^{\star}$ blue. CIA measurement values for the bee pollen samples were shown in Table 1. Mean $\mathrm{L}^{*}, \mathrm{a}^{*}$, and $\mathrm{b}^{*}$ values were $56.42,4.22,23.94$ and $56.27,4.39,25.72$ for the unpackaged and packaged bee pollen samples, respectively. Color measurement values revealed no statistically significant difference among the groups.

\subsection{Total phenolic contents and in vitro bioaccessibilities of bee pollen samples}

Total phenolic contents and in vitro bioaccessibilities of bee pollen samples were shown in Table 2. The phenolic contents of the extractable and hydrolyzable fractions and the total phenolic contents of the bee pollen samples were detected to be $16.13-74.98 \mathrm{mg} / \mathrm{g}$ GAE, $73.48-395.41 \mathrm{mg} / \mathrm{g}$ GAE, and $147.10-462.02 \mathrm{mg} / \mathrm{g} \mathrm{GAE}$, respectively. As expected, the phenolic contents of hydrolyzable fractions of all samples were higher than those of the extractable fractions. The mean values for total phenolic content (TPC) were quite high at a level of statistical significance $(\mathrm{p}<0.05)$ in the samples indicated by sample I (462.02 mg/g GAE) and J (437.55 mg/g GAE).

The total phenolic contents of bioaccessible fractions of the bee pollen samples ranged between 53.15 and $268.32 \mathrm{mg} / \mathrm{g}$ GAE. The highest value of $268.32 \mathrm{mg} / \mathrm{g}$ GAE belonged to the sample I (Table 2). The bioaccessibilities of total phenolic content were detected $36.91-77.87 \%$ for the samples. The total phenolic content bioaccessibility of the samples $\mathrm{M}(77.87 \%)$ and S(77.53\%), which sold in their original trademarked packages were significantly higher $(\mathrm{p}<0.05)$ than other samples, and followed by the other trademarked packaged products $\mathrm{P}(74.86 \%), \mathrm{T}(74.38 \%)$, R (73.39\%), and V (71.65\%) (Table 2).

Despite the insignificant differences between the packaged and unpackaged bee pollen products in terms of physicochemical properties, the total phenolic contents, antioxidant capacities, and their bioaccessibilities were quite higher at a level of statistical significance $(p<0.05)$. These differences might be explained by the packaged samples that are protected against the factors affecting the food quality such as oxidation, light, and moisture, as expected. It is known that the difference in the total phenolic

Table 2. Extractable, hydrolyzable, bioaccessible fraction and bioaccessibility (\%) of bee pollens.

\begin{tabular}{|c|c|c|c|c|c|}
\hline \multicolumn{5}{|c|}{ Total phenolic contents (mg GAE /g) } & \multirow{2}{*}{ Bioaccessibility (\%) } \\
\hline BPs & Extractable fraction & Hydrolyzable fraction & $\mathrm{TPC}^{\mathrm{a}}$ & Bioaccessible fraction & \\
\hline $\mathbf{A}^{*}$ & $47.88 \pm 2.02^{\mathrm{fg}}$ & $244.84 \pm 11.95^{\mathrm{d}}$ & $292.73 \pm 9.93^{\mathrm{d}}$ & $118.08 \pm 1.79^{\mathrm{jk}}$ & $40.37 \pm 1.98^{g}$ \\
\hline B & $45.55 \pm 3.05^{\mathrm{gh}}$ & $239.60 \pm 21.29^{\mathrm{d}}$ & $285.15 \pm 18.24^{\mathrm{de}}$ & $146.07 \pm 5.37^{\mathrm{hijk}}$ & $51.27 \pm 1.40^{\mathrm{def}}$ \\
\hline $\mathrm{C}$ & $57.52 \pm 0.58^{c}$ & $284.78 \pm 14.30^{c}$ & $342.30 \pm 13.72^{c}$ & $183.32 \pm 9.72^{\text {cdefg }}$ & $53.65 \pm 4.99^{\mathrm{de}}$ \\
\hline D & $42.00 \pm 1.31^{\mathrm{h}}$ & $282.37 \pm 19.14^{c}$ & $324.41 \pm 20.46^{c}$ & $169.74 \pm 7.21^{\mathrm{fgh}}$ & $52.50 \pm 5.53^{\mathrm{de}}$ \\
\hline E & $41.85 \pm 1.46^{\mathrm{h}}$ & $189.00 \pm 10.19^{\mathrm{e}}$ & $230.86 \pm 11.66^{\mathrm{f}}$ & $172.10 \pm 12.74^{\text {efgh }}$ & $74.50 \pm 1.76^{\mathrm{ab}}$ \\
\hline $\mathbf{F}$ & $50.2 \pm 1.13^{\mathrm{ef}}$ & $297.84 \pm 7.23^{c}$ & $348.04 \pm 8.37^{\mathfrak{c}}$ & $208.85 \pm 0.92^{\mathrm{cd}}$ & $60.03 \pm 1.71^{\mathrm{cd}}$ \\
\hline G & $25.87 \pm 1.52^{\mathrm{k}}$ & $363.59 \pm 12.22^{\mathrm{b}}$ & $389.46 \pm 13.75^{\mathrm{b}}$ & $250.81 \pm 8.52^{\mathrm{ab}}$ & $64.40 \pm 0.08^{\mathrm{bc}}$ \\
\hline $\mathbf{H}$ & $55.17 \pm 2.65^{\mathrm{cd}}$ & $241.48 \pm 11.10^{\mathrm{d}}$ & $296.65 \pm 8.45^{\mathrm{d}}$ & $151.88 \pm 6.69^{\text {ghij }}$ & $51.19 \pm 0.80^{\text {def }}$ \\
\hline I & $66.6 \pm 0.35^{\mathrm{b}}$ & $395.41 \pm 13.38^{\mathrm{a}}$ & $462.02 \pm 13.72^{\mathrm{a}}$ & $268.32 \pm 2.03^{\mathrm{a}}$ & $58.09 \pm 1.28^{\mathrm{cd}}$ \\
\hline J & $74.08 \pm 2.04^{\mathrm{a}}$ & $363.47 \pm 13.75^{\mathrm{b}}$ & $437.55 \pm 11.71^{\mathrm{a}}$ & $206.23 \pm 67.11^{\text {cde }}$ & $46.94 \pm 14.08^{\mathrm{efg}}$ \\
\hline $\mathbf{K}^{\star *}$ & $53.23 \pm 3.32^{\mathrm{de}}$ & $246.44 \pm 7.46^{\mathrm{d}}$ & $299.67 \pm 4.14^{\mathrm{d}}$ & $123.50 \pm 8.23^{\mathrm{jk}}$ & $41.20 \pm 2.18^{\mathrm{fg}}$ \\
\hline $\mathbf{L}$ & $56.14 \pm 0.49^{\mathrm{cd}}$ & $233.40 \pm 1.31^{\mathrm{d}}$ & $289.55 \pm 1.80^{\mathrm{d}}$ & $153.50 \pm 9.48^{\mathrm{ght}}$ & $53.03 \pm 3.60^{\mathrm{de}}$ \\
\hline $\mathbf{M}$ & $34.44 \pm 3.44^{j}$ & $191.37 \pm 5.83^{\mathrm{e}}$ & $225.82 \pm 2.40^{\mathrm{fg}}$ & $175.78 \pm 8.15^{\text {defgh }}$ & $77.87 \pm 4.44^{\mathrm{a}}$ \\
\hline $\mathbf{N}$ & $73.62 \pm 0.71^{\mathrm{a}}$ & $73.48 \pm 19.02^{\mathrm{f}}$ & $147.10 \pm 19.74^{\mathrm{h}}$ & $53.15 \pm 9.74^{1}$ & $36.91 \pm 11.57^{\mathrm{g}}$ \\
\hline $\mathbf{O}$ & $74.98 \pm 0.96^{\mathrm{a}}$ & $89.74 \pm 5.49^{f}$ & $164.73 \pm 6.44^{\mathrm{h}}$ & $112.40 \pm 8.32^{\mathrm{k}}$ & $68.38 \pm 7.73^{\mathrm{abc}}$ \\
\hline $\mathbf{P}$ & $38.53 \pm 2.95^{1}$ & $225.93 \pm 9.21^{\mathrm{d}}$ & $264.47 \pm 12.17^{\mathrm{e}}$ & $197.87 \pm 4.09^{\text {cdef }}$ & $74.86 \pm 1.90^{\mathrm{ab}}$ \\
\hline $\mathbf{R}$ & $41.77 \pm 1.04^{\mathrm{h} 1}$ & $167.12 \pm 9.56^{\mathrm{e}}$ & $208.89 \pm 8.52^{\mathrm{fg}}$ & $153.38 \pm 9.30^{\mathrm{ght}}$ & $73.39 \pm 1.46^{\mathrm{ab}}$ \\
\hline$S$ & $16.13 \pm 1.48^{1}$ & $186.14 \pm 14.62^{\mathrm{e}}$ & $202.27 \pm 13.14^{\mathrm{g}}$ & $156.87 \pm 11.34^{\text {ghij }}$ & $77.53 \pm 0.57^{\mathrm{a}}$ \\
\hline $\mathrm{T}$ & $42.72 \pm 0.40^{\mathrm{h}}$ & $248.23 \pm 7.07^{d}$ & $290.95 \pm 6.67^{d}$ & $216.26 \pm 8.51^{b c}$ & $74.38 \pm 4.63^{\mathrm{ab}}$ \\
\hline V & $42.38 \pm 1.63^{\mathrm{h}}$ & $243.32 \pm 6.27^{d}$ & $285.70 \pm 7.90^{\mathrm{de}}$ & $204.76 \pm 9.81^{\text {cdef }}$ & $71.65 \pm 1.45^{\mathrm{ab}}$ \\
\hline Min-Max & $16.13-74.98$ & 73.48-395.41 & $147.10-462.02$ & $53.15-268.32$ & $36.91-77.87$ \\
\hline \multicolumn{6}{|l|}{ Mean \pm SD } \\
\hline UPBp & $50.68 \pm 13.32^{\mathrm{a}}$ & $290.24 \pm 65.04^{\mathrm{a}}$ & $340.92 \pm 70.42^{\mathrm{a}}$ & $187.54 \pm 48.35^{\mathrm{a}}$ & $55.30 \pm 10.01^{b}$ \\
\hline PBp & $47.40 \pm 17.42^{\mathrm{a}}$ & $190.52 \pm 62.61^{b}$ & $237.91 \pm 54.89^{b}$ & $154.75 \pm 48.21^{\mathrm{b}}$ & $64.92 \pm 15.44^{\mathrm{a}}$ \\
\hline
\end{tabular}

Mean values (Mean) \pm standard deviation $(\mathrm{SD})(\mathrm{N}=3 \times 2)$ with different lowercase $(\mathrm{A}-\mathrm{K})$ in the same column are significantly different $(\mathrm{p}<0.05)$ according to different bee polen samples. Min: Minimum value of means, Max: Maximum values of means; BPs: Bee pollen samples; GAE: gallic acid equivalent. ${ }^{\star}$ UPBp: Unpackaged bee pollen samples A-J; ${ }^{\star \star}$ PBp: Packaged bee pollen samples K-V; ${ }^{a} \mathrm{TPC}$ : Total phenol content was calculated as the sum of extractable and hydrolyzable fraction. 
contents of the bee pollen samples might have resulted from a variety of parameters such as the growing area of plant flora, the climate, pollen harvesting procedures, methods and also the storage conditions. (Parejo et al., 2002; Žiaková et al., 2003).

\subsection{Antioxidant capacities and in vitro bioaccessibilities of the bee pollen samples}

The physiological role of antioxidants is to prevent tissue injury caused by free radicals occurring during biochemical reactions in the body (Alhan \& Şan, 2002). Antioxidant activity emerges through a series of mechanisms in which a variety of bioactive substances participate, therefore any measurement method focusing on a single group of active substances in the assessment of the antioxidant capacity of foods fails to reflect the present antioxidant capacity of the relevant food (Frankel \& Meyer, 2000; Karadag et al., 2009). Furthermore, antioxidant capacity measurements may vary actually depending on the chemical composition of food, class of the active compounds of the food, interaction of food components and active compound as well as the sample preparation technique and conditions of the antioxidant capacity method used (Sariburun et al., 2010). Therefore, the ABTS, CUPRAC, and DPPH methods were applied to assess the antioxidant capacity of the bee pollen samples taking into account the selectivity and feasibility of these methods and the data were evaluated in this study. The antioxidant capacities of extractable, hydrolyzable and bioaccessible fractions according to these three methods were given in Table 3 .

According to the results of the ABTS method, antioxidant capacities of extractable fractions of the bee pollen samples ranged between 6.20 and $38.20 \mu \mathrm{mol} \mathrm{TE} / \mathrm{g}$, whereas those of hydrolyzable fractions were 37.63-80.49 $\mu \mathrm{mol} \mathrm{TE} / \mathrm{g}$ (Table 3). The highest value $(111.40 \mu \mathrm{mol} \mathrm{TE} / \mathrm{g})$ for antioxidant capacity belonged to the sample $\mathrm{D}$, followed by the samples $\mathrm{F}$ $(110.22 \mu \mathrm{mol} \mathrm{TE} / \mathrm{g})$ and $\mathrm{C}(99.40 \mu \mathrm{mol} \mathrm{TE} / \mathrm{g})$. The mean value of total antioxidant capacity (TAC) of the unpackaged bee pollen samples, which was determined to be $89.13 \mu \mathrm{mol} \mathrm{TE} / \mathrm{g}$ was higher than packaged products $(74.19 \mu \mathrm{mol} \mathrm{TE} / \mathrm{g})$, unexpected. According to the CUPRAC method, the highest TAC value of $257.27 \mu \mathrm{mol} \mathrm{TE} / \mathrm{g}$ was detected in the sample $\mathrm{N}$, followed by the samples P $(242.37 \mu \mathrm{mol} \mathrm{TE} / \mathrm{g}), \mathrm{O}(207.43 \mu \mathrm{mol} \mathrm{TE} / \mathrm{g})$, and $\mathrm{L}(202.84 \mu \mathrm{mol} \mathrm{TE} / \mathrm{g})$, respectively. The highest values in terms of the total antioxidant capacities of both fractions were obtained with the CUPRAC method in comparison to the other methods used. This situation might be explained by CUPRAC method have sensitivity and selectivity for both hydrophilic and lipophilic materials and also the feasibility of the method for the bioactive compounds in bee pollen samples (Apak et al., 2004).

When the antioxidant capacities of the bee pollen samples were measured by the DPPH method the hydrolyzable fractions (33.21-62.37 $\mu \mathrm{mol} \mathrm{TE} / \mathrm{g}$ ) exhibited significantly higher values than

Table 3. Extractable, hydrolyzable and bioaccessible antioxidant capacities of bee pollens.

\begin{tabular}{|c|c|c|c|c|c|c|c|c|c|}
\hline \multicolumn{10}{|c|}{ Antioxidant capacity $(\mu \mathrm{mol} \mathrm{TE} / \mathrm{g})$} \\
\hline \multirow[b]{2}{*}{ BPs } & \multicolumn{3}{|c|}{ ABTS } & \multicolumn{3}{|c|}{ CUPRAC } & \multicolumn{3}{|c|}{ DPPH } \\
\hline & $\begin{array}{l}\text { Extractable } \\
\text { fraction }\end{array}$ & $\begin{array}{l}\text { Hyrolysable } \\
\text { fraction }\end{array}$ & Total AC & $\begin{array}{l}\text { Extractable } \\
\text { fraction }\end{array}$ & $\begin{array}{l}\text { Hydrolysable } \\
\text { fraction }\end{array}$ & Total AC & $\begin{array}{l}\text { Extractable } \\
\text { fraction }\end{array}$ & $\begin{array}{l}\text { Hydrolysable } \\
\text { fraction }\end{array}$ & Total AC \\
\hline B & $16.56 \pm 0.28^{\mathrm{h}}$ & $61.30 \pm 3.64^{\text {cde }}$ & $77.87 \pm 3.36^{\mathrm{fg}}$ & $21.51 \pm 0.55^{\mathrm{ef}}$ & $83.55 \pm 1.49^{\mathrm{h}_{1}}$ & $105.07 \pm 0.98^{\mathrm{ijk}}$ & $10.83 \pm 0.13^{j}$ & $62.37 \pm 0.68^{\mathrm{a}}$ & $73.20 \pm 0.55^{c}$ \\
\hline $\mathrm{C}$ & $35.21 \pm 0.26^{\mathrm{b}}$ & $64.18 \pm 1.74^{c}$ & $99.40 \pm 2.01^{\mathrm{b}}$ & $15.65 \pm 4.92^{\mathrm{fg}}$ & $93.76 \pm 0.76^{g}$ & $109.41 \pm 4.16^{1}$ & $16.26 \pm 0.18^{\mathrm{d}}$ & $50.61 \pm 0.79^{d}$ & $66.87 \pm 0.61^{\mathrm{d}}$ \\
\hline $\mathbf{D}$ & $30.90 \pm 0.30^{c}$ & $80.49 \pm 3.30^{\mathrm{a}}$ & $111.40 \pm 3.61^{\mathrm{a}}$ & $47.96 \pm 0.48^{\mathrm{b}}$ & $79.25 \pm 0.79^{i j}$ & $127.22 \pm 0.30^{\mathrm{h}}$ & $13.70 \pm 0.28^{\mathrm{fg}}$ & $41.63 \pm 1.39$ & $55.33 \pm 1.12^{\mathrm{g}}$ \\
\hline F & $37.99 \pm 0.30^{\mathrm{a}}$ & $72.22 \pm 1.28^{\mathrm{b}}$ & $110.22 \pm 0.98^{\mathrm{a}}$ & $26.08 \pm 2.95^{\mathrm{de}}$ & $136.91 \pm 2.77^{\mathrm{e}}$ & $163.01 \pm 5.73^{\mathrm{e}}$ & $20.69 \pm 0.70^{\mathrm{b}}$ & $54.11 \pm 0.47^{\mathrm{c}}$ & $74.80 \pm 1.17^{\mathrm{c}}$ \\
\hline G & $16.62 \pm 0.63^{\mathrm{h}}$ & $57.95 \pm 0.90^{\mathrm{ef}}$ & $74.58 \pm 0.27^{\mathrm{gh}}$ & $8.32 \pm 1.46^{\mathrm{gh}}$ & $139.62 \pm 0.88^{\mathrm{e}}$ & $147.95 \pm 0.58^{\mathrm{g}}$ & $12.47 \pm 0.24^{1}$ & $42.21 \pm 1.42^{\mathrm{f}}$ & $54.68 \pm 1.66^{\mathrm{g}}$ \\
\hline $\mathbf{H}$ & $37.87 \pm 0.13^{\mathrm{a}}$ & $50.69 \pm 1.99^{\mathrm{gh}}$ & $88.57 \pm 1.85^{\text {cde }}$ & $8.68 \pm 1.96^{\mathrm{gh}}$ & $183.90 \pm 4.26^{\mathrm{b}}$ & $192.58 \pm 6.23^{\mathrm{d}}$ & $16.50 \pm 0.18^{d}$ & $44.31 \pm 0.94^{\mathrm{e}}$ & $60.81 \pm 0.76^{\text {ef }}$ \\
\hline I & $38.20 \pm 0.10^{\mathrm{a}}$ & $53.12 \pm 0.54^{\mathrm{gh}}$ & $91.33 \pm 0.45^{\mathrm{cd}}$ & $6.59 \pm 3.93^{\mathrm{h}}$ & $152.50 \pm 1.64^{\mathrm{d}}$ & $159.10 \pm 5.57^{\mathrm{ef}}$ & $19.44 \pm 0.18^{c}$ & $40.39 \pm 0.91^{\mathrm{fgh}}$ & $59.83 \pm 1.08^{\mathrm{f}}$ \\
\hline J & $35.32 \pm 0.40^{\mathrm{b}}$ & $49.98 \pm 1.70^{\mathrm{h}}$ & $85.32 \pm 1.30^{\mathrm{e}}$ & $11.13 \pm 3.44^{\mathrm{gh}}$ & $137.23 \pm 1.51^{\mathrm{e}}$ & $148.37 \pm 1.94^{\mathrm{g}}$ & $15.65 \pm 0.24^{\mathrm{e}}$ & $39.16 \pm 0.33^{\mathrm{hr}}$ & $54.81 \pm 0.57^{\mathrm{g}}$ \\
\hline $\mathbf{K}^{* *}$ & $17.30 \pm 0.75^{\mathrm{h}}$ & $51.78 \pm 0.08^{\mathrm{gh}}$ & $69.09 \pm 0.67^{1}$ & $14.57 \pm 2.47^{\mathrm{fg}}$ & $138.82 \pm 4.48^{\mathrm{e}}$ & $153.41 \pm 6.95^{\mathrm{fg}}$ & $2.76 \pm 0.09^{1}$ & $41.68 \pm 0.57^{\mathrm{fg}}$ & $44.43 \pm 0.66^{1}$ \\
\hline O & $20.76 \pm 0.75^{\mathrm{f}}$ & $58.68 \pm 0.65^{\mathrm{e}}$ & $79.45 \pm 0.10^{\mathrm{f}}$ & $33.32 \pm 3.47^{\mathrm{cd}}$ & $174.10 \pm 7.25^{c}$ & $207.43 \pm 10.72^{c}$ & $0.44 \pm 0.05^{\mathrm{m}}$ & $35.25 \pm 1.12 \mathrm{jk}$ & $35.69 \pm 1.16^{j}$ \\
\hline $\mathbf{P}$ & $14.67 \pm 0.23^{1}$ & $72.68 \pm 1.03^{\mathrm{b}}$ & $87.36 \pm 1.27^{\mathrm{de}}$ & $57.70 \pm 0.39^{\mathrm{a}}$ & $184.66 \pm 3.05^{\mathrm{b}}$ & $242.37 \pm 2.66^{\mathrm{b}}$ & $22.45 \pm 0.38^{\mathrm{a}}$ & $61.39 \pm 2.06^{\mathrm{a}}$ & $83.84 \pm 2.44^{\mathrm{a}}$ \\
\hline $\mathbf{R}$ & $10.07 \pm 0.55^{\mathrm{k}}$ & $43.01 \pm 1.43^{1}$ & $53.09 \pm 1.99^{k}$ & $19.45 \pm 3.44^{\mathrm{ef}}$ & $86.99 \pm 2.36^{\mathrm{h}}$ & $106.44 \pm 5.80^{i j}$ & $13.40 \pm 0.14^{\mathrm{fgh}}$ & $33.21 \pm 1.23^{\mathrm{k}}$ & $46.61 \pm 1.37^{1}$ \\
\hline S & $6.20 \pm 0.78^{1}$ & $54.02 \pm 2.22^{\mathrm{fg}}$ & $60.23 \pm 3.01^{j}$ & $38.18 \pm 2.50^{c}$ & $116.34 \pm 2.52^{\mathrm{f}}$ & $154.53 \pm 5.03^{\mathrm{efg}}$ & $13.91 \pm 0.25^{\mathrm{f}}$ & $54.76 \pm 1.31^{\mathrm{c}}$ & $68.67 \pm 1.56^{\mathrm{d}}$ \\
\hline $\mathrm{T}$ & $22.28 \pm 0.29^{\mathrm{e}}$ & $64.93 \pm 0.75^{c}$ & $87.22 \pm 0.46^{\mathrm{de}}$ & $24.35 \pm 8.35^{\mathrm{e}}$ & $73.22 \pm 5.54^{\mathrm{j} k}$ & $97.58 \pm 2.81^{\mathrm{jk}}$ & $20.18 \pm 0.04^{\mathrm{b}}$ & $57.31 \pm 0.65^{\mathrm{b}}$ & $77.48 \pm 0.61^{\mathrm{b}}$ \\
\hline V & $11.32 \pm 0.48^{j}$ & $37.63 \pm 1.18^{j}$ & $48.96 \pm 1.67^{\mathrm{k}}$ & $6.25 \pm 3.45^{\mathrm{h}}$ & $76.98 \pm 4.49^{j}$ & $83.24 \pm 1.04^{1}$ & $15.50 \pm 0.55^{\mathrm{e}}$ & $40.08 \pm 0.58^{\mathrm{gh}}$ & $55.58 \pm 1.13^{\mathrm{g}}$ \\
\hline Min-Max & $6.20-38.20$ & $37.63-80.49$ & 48.96-111.40 & $6.25-64.88$ & 69.16-192.96 & 83.24-257.27 & $0.44-22.45$ & $33.21-62.37$ & $35.69-83.84$ \\
\hline \multicolumn{10}{|l|}{ Mean \pm SD } \\
\hline UPBp & $31.30 \pm 8.40^{\mathrm{a}}$ & $57.83 \pm 11.76^{a}$ & $89.13 \pm 13.74^{\mathrm{a}}$ & $20.56 \pm 13.59^{\mathrm{a}}$ & $115.14 \pm 38.56^{\mathrm{a}}$ & $135.71 \pm 30.64^{b}$ & $15.17 \pm 3.07^{\mathrm{a}}$ & $45.33 \pm 7.73^{\mathrm{a}}$ & $60.50 \pm 8.25^{\mathrm{a}}$ \\
\hline PBp & $17.05 \pm 6.18^{b}$ & $57.14 \pm 10.83^{\mathrm{a}}$ & $74.19 \pm 15.17^{b}$ & $38.45 \pm 21.93^{b}$ & $131.88 \pm 42.55^{\mathrm{a}}$ & $170.33 \pm 59.47^{\mathrm{a}}$ & $10.70 \pm 7.25^{b}$ & $46.85 \pm 9.59^{\mathrm{a}}$ & $57.55 \pm 14.92^{\mathrm{a}}$ \\
\hline
\end{tabular}

Mean values represented by the same letters within the same column are not significantly different at $\mathrm{p} \leq 0.05$. Data are expressed as means \pm standard deviations (SD). Min: Minimum value of means, Max: Maximum values of means. BPs: Bee pollen samples; TE: Trolox Equivalent. ${ }^{\star} \mathrm{UPBp}$ : Unpackaged bee pollen samples A-J; ${ }^{* *} \mathrm{PBp}$ : Packaged bee pollen samples K-V. 
those of extractable fractions $(0.44-22.45 \mu \mathrm{mol} \mathrm{TE} / \mathrm{g})$. No significant differences in total antioxidant capacity levels measured by the DPPH method were found between packaged and unpackaged bee pollen samples $(p>0.05)$. The lowest antioxidant capacity value has obtained in this method when compared to the other methods used. Therefore, it can be concluded that the DPPH method is not suitable for the assessment of the antioxidant capacity of bee pollen.

Bee pollen is a biological substance covered with a double-layered wall. The outer layer called exine is composed of sporopollenin and the inner layer intine has a semipermeable membrane-like structure. The exine is a complex solid substance that is found to have survived on the surfaces of fossils of millions of years of age. It is rather hard and even impossible to digest the material on most occasions (Frenguelli, 2003). Therefore, the extractable fractions have lower antioxidant capacity than the hydrolyzable fractions in all of the antioxidant capacity methods used.

Previous research showed that bee pollen had a high antioxidant capacity (Almedia et al., 2017; Campos et al., 2003; Suriyatem et al., 2017; Sun et al., 2017; Mărghitaş et al. 2009; Carpes et al., 2007) that varies due to the ecological diversity and predominance of the plant species that it was collected from. However, there is no direct correlation available between the total phenolic content (TPC) and the antioxidant capacity (Özkök \& Silici, 2017; Dorman et al., 2003; Miliauskas et al., 2004). TPC of 12 bee pollen samples were determined to be $691.67-1383.67 \mathrm{mg} / \mathrm{kg}$ in a study conducted in Slovakia (Fatrcová-Šramková et al., 2013) Özkök \& Silici (2017) reported in a study performed with 20 bee pollens that the mean total phenolic content of samples was $2340.07 \mathrm{mg}$ GAE/100 g and the mean antioxidant capacities were $42.37 \mathrm{mg}$ AAE/g. Yesiltas et al. (2014) indicated that total phenolic content of 9 bee pollen samples collected from different regions of Turkey was 12.0-36.7 mg GAE/g) and antioxidant capacities of the samples according to ABTS, CUPRAC, DPPH, and FRAP methods were 15.2-33.6 mg TE/g, 20.7-89.4 mg TE/g, 5.7-15.2 mg TE/g, and 5.2-15.7 mg TE/g, respectively. Karkar et al. (2018) reported that antioxidant capacities of chestnut bee pollen samples were 3.70-34.18 mg TE/g, 3.15-20.24 mM TE/g by CHROMAC ve FRAP methods, respectively. A previous study by Anjos et al., (2019a) average total phenolic content of 6 bee pollen samples which found as predominant pollen Cistus ladanifer (42.6\%) were detected $6.81 \mathrm{mg} \mathrm{QE} / \mathrm{g}$ and average antioxidant capacity was EC50: $2.62 \mathrm{mg} / \mathrm{mL}$ by DPPH methods. On the other hand, the total phenolic content value (6.9-21 mg GAE/g) in bee pollens were lower than the values in this study reported by Duarte et al. (2018). Also, Duarte et al. (2018) declared that bee pollen contains T. clavipes (123.4 mg GAE/100) were the highest antioxidant capacity using FRAP assay and the highest correlation (0.98) between FRAP assay and total phenolic content.

The widely ranging results of the above-mentioned studies were linked with the climate changes, the origin of the harvested plant as well as the storage conditions of the products (Anjos et al., 2019b; Choi et al., 2006; Kumazawa et al., 2004; Marcucci et al., 2001; Almaraz-Abarca et al., 2004). Also, the chemical and nutritional quality of bee pollens depend on the harvest and transport methods, storage conditions and packaging properties (Sattler et al., 2015; Arruda et al., 2013).
Bioaccessibility of antioxidants \%

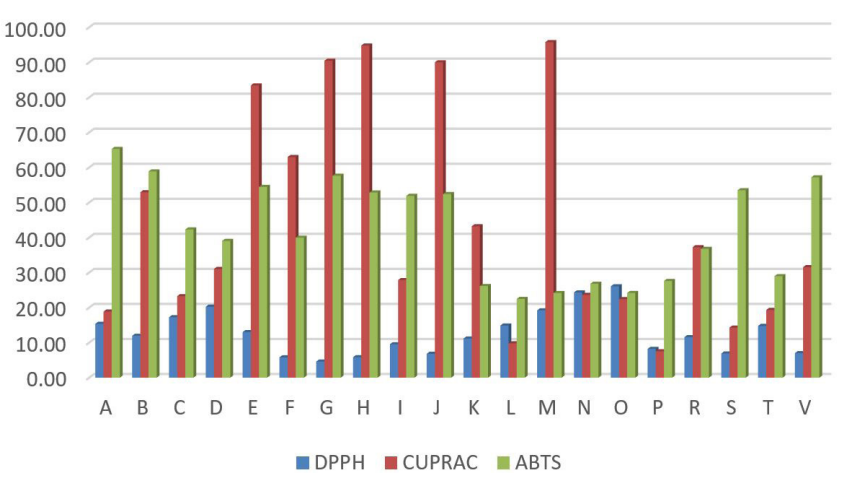

Figure 1. Bioaccessibility (\%) of antioxidant capacities of bee pollen.

The absorbed amount of a nutrient in the gastrointestinal tract known as bioaccessibility may vary depending on numerous factors such as the physical structure and chemical composition of the food, interactions of the food ingredients, and the processing methods (Sandström, 2001). In vitro bioaccessibilities of the bee pollen samples included in the study, which were assessed by three different antioxidant capacity measurement methods (ABTS, CUPRAC and DPPH) were shown in Figure 1. Bioaccessibility values were detected to be 7.54-95.76\%, 22.47-65.28, and 4.59-26.07 by CUPRAC, ABTS, and DPPH methods, respectively. These differences between antioxidant methods might be the diversity in the chemical composition of the bee pollen samples and the sensibility of each method toward different bioactive compounds (Al-İsmail \& Aburjai, 2004; Almeida-Muradian et al., 2005; Morais et al., 2011). In the presented study, CUPRAC method proved to be the most appropriate method for bioaccessibility, which was considered to have resulted from the high sensibility of the active compounds with high antioxidant capacities to the relevant method.

Based on overall evaluations, average values for AC bioaccessibilities of the unpackaged products (A-J) were determined to be $57.56 \%, 50.33 \%$ and $11.04 \%$, according to CUPRAC, ABTS, and DPPH methods, whereas those of the packaged bee pollen products (K-V) were $30.48 \%, 32.49 \%$, and $14.41 \%$, respectively (Figure 1).

\section{Conclusion}

Bee pollen is considered a functional food product with its high total phenolic content and antioxidant capacity. Besides, the bioaccessibilities of the antioxidant capacity was determined to be fairly high. Based on the results of all antioxidant capacity assays used in the study, it was demonstrated that the antioxidant capacities of the hydrolyzable fractions were higher than those of the extractable fractions. Furthermore, the CUPRAC method proved to be significantly superior $(\mathrm{p}<0.05)$ to ABTS and DPPH methods based on the data obtained through the assessments of TAC and their bioaccessibilities. The difference between the packaged and unpackaged bee pollen products in terms of antioxidant properties is considered highly likely to be associated with the chemical composition, harvested plant species, geographical features and the climate of the harvesting area, and 
certain environmental circumstances as well as the storage and sales conditions of the bee pollen products. Preservation of the chemical compounds such as antioxidants present in bee pollens depends on good storage conditions. Antioxidant properties because of phenolics are decreased based on storage conditions contained high heat, light, oxygen or moisture during shelf life. For this reason, food packaging of bee pollen positively affected antioxidant properties in this study. Therefore, bee pollen should be stored in a cool, dry place and in well packaging materials. Consequently, it can be deduced that bee pollen with its general chemical features has emerged as a natural antioxidant source in a healthy diet both solely and as a supplement contained in different food products.

\section{References}

Alhan, C. C., \& Şan, M. (2002). Are the anti-oxidants helpful in therapy of patients with coronary heart disease? Turkish Clinical Journal Cardiology, 15, 203-213.

Alicic, D., Subaric, D., Jasic, M., Pasalic, H., \& Ackar, D. (2014). Antioksidant properties of pollen. Hrana u Zdravlju i Bolesti: Znanstveno-stručni Časopis za Nutricionizam i Dijetetiku, 3(1), 6-12.

Al-İsmail, K. M., \& Aburjai, T. A. (2004). Antioxidant activity of water and alcohol extracts of chamomile flowers, anise seeds and dill seeds. Journal of the Science of Food and Agriculture, 84(2), 173-178. http:// dx.doi.org/10.1002/jsfa.1625.

Almaraz-Abarca, N., Campos, M. G., Ávila-Reyes, J. A., Naranjo-Jiménez, N., Herrera-Corral, J., \& González-Valdez, L. S. (2004). Variability of antioxidant activity among honeybee-collected pollen of different botanical origin. Interciencia, 29(10), 574-578.

Almeida, J. D., Reis, A. S., Heldt, L. F., Pereira, D. K., Bianchin, M., Moura, C. D., Plata-Oviedo, M. S., Haminiuk, C. W., Ribeiro, I. S., Luz, C. F., \& Carpes, S. T. (2017). Lyophilized bee pollen extract: A natural antioxidant source to prevent lipid oxidation in refrigerated sausages. Lebensmittel-Wissenschaft + Technologie, 76, 299-305. http://dx.doi.org/10.1016/j.lwt.2016.06.017.

Almeida-Muradian, L. B., Pamplona, L. C., Coimbra, S., \& Barth, O. M. (2005). Chemical composition and botanical evaluation of dried bee pollen pellets. Journal of Food Composition and Analysis, 18(1), 105-111. http://dx.doi.org/10.1016/j.jfca.2003.10.008.

Anjos, O., Fernandes, R., Cardoso, S. M., Delgado, T., Farinha, N., Paula, V., Estevinho, L. M., \& Carpes, S. T. (2019a). Bee pollen as a natural antioxidant source to prevent lipid oxidation in black pudding. Lebensmittel-Wissenschaft + Technologie, 111, 869-875. http://dx.doi.org/10.1016/j.lwt.2019.05.105.

Anjos, O., Paula, V., Delgado, T., \& Estevinho, L. M. (2019b). Influence of the storage conditions on the quality of bee pollen. ZemdirbysteAgriculture, 106(1), 87-94. http://dx.doi.org/10.13080/z-a.2019.106.012.

Apak, R., Güclü, K., Özyürek, M., \& Karademir, S. E. (2004). Novel total antioxidant capacity index for dietary polyphenols and vitamins $\mathrm{C}$ and $\mathrm{E}$, using their cupric ion reducing capability in the presence of neocuproine: CUPRAC method. Journal of Agricultural and Food Chemistry, 52(26), 7970-7981. http://dx.doi.org/10.1021/jf048741x. PMid:15612784.

Apak, R., Güclü, K., Özyürek, M., \& Çelik, S. E. (2008). Mechanism of antioxidant capacity assays and the CUPRAC (cupric ion reducing antioxidant capacity) assay. Mikrochimica Acta, 160(4), 413-419. http://dx.doi.org/10.1007/s00604-007-0777-0.

Arruda, V. A. S., Santos Pereira, A. A., Estevinho, L. M., \& AlmeidaMuradian, L. B. (2013). Presence and stability of B complex vitamins in bee pollen using different storage conditions. Food and Chemical Toxicology, 51, 143-148. http://dx.doi.org/10.1016/j.fct.2012.09.019. PMid:23022013.

Association of Official Analytical Chemists - AOAC. (2000). Official methods of analysis (17th ed.). Maryland: AOAC.

Bárbara, M. S., Machado, C. S., Sodré, G. S., Dias, L. G., Estevinho, L. M., \& Carvalho, C. A. (2015). Microbiological assessment, nutritional characterization and phenolic compounds of bee pollen from Mellipona mandacaia Smith, 1983. Molecules, 20(7), 12525-12544. http://dx.doi.org/10.3390/molecules200712525.

Bogdanov, S. (2006). Contaminants of bee products. Apidologia, 37(1), 1-18. http://dx.doi.org/10.1051/apido:2005043.

Bonvehí, J. S., Torrentó, M. S., \& Lorente, E. C. (2001). Evaluation of polyphenolic and flavonoid compounds in honeybee-collected pollen produced in Spain. Journal of Agricultural and Food Chemistry, 49(4), 1848-1853. http://dx.doi.org/10.1021/jf0012300. PMid:11308335.

Borycka, K., Grabek-Lejko, D., \& Kasprzyk, I. (2015). Antioksidant and antibacterial properties of commercial bee pollen prooducts. Journal of Apicultural Research, 54(5), 491-502. http://dx.doi.org/1 0.1080/00218839.2016.1185309.

Brand-Williams, W., Cuvelier, M. E., \& Berset, C. (1995). Use of a free radical method to evaluate antioxidant activity. LebensmittelWissenschaft + Technologie, 28(1), 25-30. http://dx.doi.org/10.1016/ S0023-6438(95)80008-5.

Brasil, Ministério de Agricultura e do Abastecimento. (2001, January 23). Regulamentos técnicos de identidade e qualidade de apitoxina, cera de abelha, geléia real, geléia real liofilizada, pólen apícola, própolis e extrato de própolis (Instrução normativa no 3, de 19 de janeiro de 2001). Diário Oficial [da] República Federativa do Brasil, seção 1. Retrieved from http://www.extranet.agricultura.com.br

Campos, M. G., Webby, R. F., Markham, K. R., Mitchell, K. A., \& da Cunha, A. P. (2003). Age-induced diminution of free radical scavenging capacity in bee pollens and the contribution of constituent flavonoids. Journal of Agricultural and Food Chemistry, 51(3), 742-745. http:// dx.doi.org/10.1021/jf0206466. PMid:12537451.

Carpes, S. T., Begnini, R., Alencar, S. M., \& Masson, M. L. (2007). Study of preparations of bee pollen extracts, antioksidant and antibacterial activity. Ciência e Agrotecnologia, 31(6), 1818-1825. http://dx.doi. org/10.1590/S1413-70542007000600032.

Čeksteryté, V., Kurtinaitienė, B., Venskutonis, P. R., Pukalskas, A., Kazernavičiūtė, R., \& Balžekas, J. (2016). Evaluation of antioksidant activity and flavonoid composition in differently preserved bee products. Czech Journal of Food Sciences, 34(2), 133-142. http:// dx.doi.org/10.17221/312/2015-CJFS.

Choi, Y. M., Noh, D. O., Cho, S. Y., Suh, H. J., Kim, K. M., \& Kim, J. M. (2006). Antioxidant and antimicrobial activities of propolis from several regions of Korea. Lebensmittel-Wissenschaft + Technologie, 39(7), 756-761. http://dx.doi.org/10.1016/j.lwt.2005.05.015.

Coronel, B. B., Grasso, D. S. C., Pereira, G., \& Fernández, A. (2004). Caracterización bromatológica del polen apícola argentino. Ciencia, Docencia y Tecnología, 29, 145-181. Retrieved from http://www. redalyc.org/articulo.oa?id=14502906

Dorman, H. J. D., Peltoketo, A., Hiltunen, R., \& Tikkanen, M. J. (2003). Characterization of the antioxidant properties of de-odorized aqueous extracts from selected Lamiaceae herbs. Food Chemistry, 83(2), 255-262. http://dx.doi.org/10.1016/S0308-8146(03)00088-8.

Duarte, A. W., Vasconcelos, M. R., Oda-Souza, M., Oliveira, F. F., \& López, A. M. (2018). Honey and bee pollen produced by Meliponini (Apidae) in Alagoas, Brazil: multivariate analysis of physicochemical 
and antioxidant profiles. Food Science and Technology, 38(3), 493503. http://dx.doi.org/10.1590/fst.09317.

Fadzilah, N. H., Jaapar, M. F., Jajuli, R., \& Omar, W. A. W. (2017). Total phenolic content, total flavonoid and antioksidant activity of ethanolic bee pollen extracts from three species of Malaysian stingless bee. Journal of Apicultural Research, 56(2), 130-135. http://dx.doi.org/1 0.1080/00218839.2017.1287996.

Fatrcová-Šramková, K., Nôžková, J., Kačániová, M., Máriássyová, M., Rovná, K., \& Stričík, M. (2013). Antioxidant and antimicrobial properties of monofloral bee pollen. Journal of Environmental Science and Health. Part. B, Pesticides, Food Contaminants, and Agricultural Wastes, 48(2), 133-138. http://dx.doi.org/10.1080/03601234.2013.7 27664. PMid:23305281.

Feás, X., Vázquez-Tato, M. P., Estevinho, L., Seijas, J. A., \& Iglesias, A. (2012). Organic bee pollen: botanical origin, nutritional value, bioactive compounds, antioxidant activity and microbiological quality. Molecules, 17(7), 8359-8377. http://dx.doi.org/10.3390/ molecules17078359. PMid:22785265.

Frankel, E. N., \& Meyer, A. S. (2000). The problems of using one-dimensional methods to evaluate multifunctional food and biological antioxidants. Journal of the Science of Food and Agriculture, 80(13), 1925-1941. http://dx.doi.org/10.1002/1097-0010(200010)80:13<1925::AIDJSFA714>3.0.CO;2-4.

Frenguelli, G. (2003). Pollen structure and morphology. Postepy Dermatologii i Alergologii, 20(4), 200-204.

Guiné, R. P. F. (2015). Bee pollen: chemical composition and potential beneficial effects on health. Current Nutrition and Food Science, 11(4), 301-308. http://dx.doi.org/10.2174/1573401311666150630181615.

Isık, A., Ozdemir, M., \& Doymaz, İ. (2019). Effect of hot air drying on quality characteristics and physicochemical properties of bee pollen. Food Science and Technology, 39(1), 224-231. http://dx.doi. org/10.1590/fst.02818.

Kacániová, M., Vukovic, N., Chlebo, R., Hascík, P., Rovná, K., Cubon, J., Dzugan, M., \& Pasternakiewicz, A. (2012). The antimicrobial activity of honey, bee pllen loads and beeswax from Slovakia. Archives of Biological Sciences, 64(3), 927-934. http://dx.doi.org/10.2298/ ABS1203927K.

Karadag, A., Ozçelik, B., \& Saner, S. (2009). Review of methods to determine antioxidant capacities. Food Analytical Methods, 2(1), 41-60. http://dx.doi.org/10.1007/s12161-008-9067-7.

Karadal, F., Ertaş Onmaz, N., Abay, S., Yıldırım, Y., Al, S., Tatyuz, I., \& Akçay, A. (2018). A study of antibacteral and antioksidant activities of bee products: propolis, pollen and honey samples. The Ethiopian Journal of Health Development, 32(2), 116-122.

Karkar, B., Şahin, S., \& Güneş, M. E. (2018). Antioxidative effect of Turkish chestnut bee pollen on DNA oxidation system and its phenolic compounds. The Journal of Food, 43(1), 34-42. http:// dx.doi.org/10.15237/gida.GD17055.

Komosinska-Vassev, K., Olczyk, P., Kazmierczak, J., Mencner, L., \& Olczyk, K. (2015). Bee pollen: chemical composition and therapeutic application. Evidence-Based Complementary and Alternative Medicine, 2015, 297425. http://dx.doi.org/10.1155/2015/297425. PMid:25861358.

Kumazawa, S., Hamasaka, T., \& Nakayama, T. (2004). Antioxidant activity of propolis of various geographic origins. Food Chemistry, 84(3), 329-339. http://dx.doi.org/10.1016/S0308-8146(03)00216-4.

Marchini, L. C., Reis, V. D. A., \& Moreti, A. C. C. C. (2006). Composição físico-química de amostras de pólen coletado por abelhas Africanizadas Apis mellifera (Hymenoptera:Apidae) em Piracicaba, Estado de São Paulo. Ciência Rural, 36(3), 949-953. http://dx.doi.org/10.1590/ S0103-84782006000300034.
Marcucci, M. C., Ferreres, F., García-Viguera, C., Bankova, V. S., Castro, S. L., Dantas, A. P., Valente, P. H., \& Paulino, N. (2001). Phenolic compounds from Brazilian propolis with pharmacological activities. Journal of Ethnopharmacology, 74(2), 105-112. http://dx.doi. org/10.1016/S0378-8741(00)00326-3. PMid:11167028.

Mărghitaş, L. A., Stanciu, O. G., Dezmirean, D. S., Bobiş, O., Popescu, O., Bogdanov, S., \& Campos, M. G. (2009). In vitro antioxidant capacity of honeybee-collected pollen of selected floral origin harvested from Romania. Food Chemistry, 115(3), 878-883. http:// dx.doi.org/10.1016/j.foodchem.2009.01.014.

Melo, I. L. P., \& Almeida-Muradian, L. B. (2011). Comparison of methodologies for moisture determination on dried bee pollen samples. Food Science and Technology-. Food Science and Technology, 31(1), 194-197. http://dx.doi.org/10.1590/S0101-20612011000100029.

Miliauskas, G., Venskutonis, P. R., \& van Beek, T. A. (2004). Screening of radical scavenging activity of some medicinal and aromatic plant extracts. Food Chemistry, 85(2), 231-237. http://dx.doi.org/10.1016/j. foodchem.2003.05.007.

Morais, M., Moreira, L., Feas, X., \& Estevinho, L. M. (2011). Honeybeecollected pollen from five Portuguese Natural Parks: Palynological origin, phenolic content, antioxidant properties and antimicrobial activity. Food and Chemical Toxicology, 49(5), 1096-1101. http:// dx.doi.org/10.1016/j.fct.2011.01.020. PMid:21291944.

Naczk, M., \& Shahidi, F. (2004). Extraction and analysis of phenolics in food. Journal of Chromatography. A, 1054(1-2), 95-111. http:// dx.doi.org/10.1016/S0021-9673(04)01409-8. PMid:15553136.

Özkök, D., \& Silici, S. (2017). Antioksidant activities of honeybee products and their mixtures. Food Science and Biotechnology, 26(1), 201-206. http://dx.doi.org/10.1007/s10068-017-0027-0. PMid:30263529.

Parejo, I., Viladomat, F., Bastida, J., Rosas-Romero, A., Flerlage, N., Burillo, J., \& Codina, C. (2002). Comparison between the radical scavenging activity and antioxidant activity of six distilled and nondistiled Mediterranean herbs and aromatic plants. Journal of Agricultural and Food Chemistry, 50(23), 6882-6890. http://dx.doi. org/10.1021/jf020540a. PMid:12405792.

Pascoal, A., Rodrigues, S., Teixeira, A., Feas, X., \& Estevinho, L. M. (2014). Biological activities of commercial bee pollens: Antimicrobial, antimutagenic, antioksidant and anti-inflammatory. Food and Chemical Toxicology, 63, 233-239. http://dx.doi.org/10.1016/j. fct.2013.11.010. PMid:24262487.

Rzepecka-Stojko, A., Stojko, J., Kurek-Gorecka, A., Gorecki, M., Sobczak, A., Stojko, R., \& Buszman, E. (2015). Polyphenol content and antioksidant activity of bee pollen extracts from Poland. Journal of Apicultural Research, 54(5), 482-490. http://dx.doi.org/10.1080/ 00218839.2016 .1186916$.

Sandıkçı Altunatmaz, S., Tarhan, D., Aksu, F., Barutçu, Ü. B., \& Or, M. E. (2017). Mineral element and heavy metal (cadmium, lead and arsenic) leveles of bee pollen in Turkey. Food Science and Technology, 37(Suppl. 1), 136-141. http://dx.doi.org/10.1590/1678-457x.36016.

Sandström, B. (2001). Micronutrient interactions: effects on absorption and bioavailability. British Journal of Nutrition, 85(2, Suppl. 2), 181-185. http://dx.doi.org/10.1079/BJN2000312. PMid:11509108.

Sariburun, E., Sahin, S., Demir, C., Türkben, C., \& Uylaser, V. (2010). Phenolic content and antioxidant activity of raspberry and blackberry cultivars. Journal of Food Science, 75(4), 328-335. http://dx.doi. org/10.1111/j.1750-3841.2010.01571.x. PMid:20546390.

SAS Institute Inc. - SAS. (2007). Statistical discovery from SAS. Cary: SAS.

Sattler, J. A., Melo, I. L., Granato, D., Araújo, E. D., Freitas, A. D., Barth, O. M., Sattler, A., \& Almeida-Muradian, L. B. (2015). Impact of origin on bioactive compounds and nutritional composition of bee pollen from southern Brazil: a screening study. Food Research International, 77, 82-91. http://dx.doi.org/10.1016/j.foodres.2015.09.013. 
Sun, L., Guo, Y., Zhang, Y., \& Zhuang, Y. (2017). Antioxidant and anti-tyrosinase activities of phenolic extracts from rape bee pollen and inhibitory melanogenesis by cAMP/ MITF/TYR Pathway in B16 mouse melanoma cells. Frontiers in Pharmacology, 8(104), 1-9. http://dx.doi.org/10.3389/ fphar.2017.00104. PMid:28337140.

Suriyatem, R., Auras, R. A., Intipunya, P., \& Rachtanapun, P. (2017). Predictive mathematical modeling for EC50 calculation of antioxidant activity and antibacterial ability of Thai bee products. Journal of Applied Pharmaceutical Science, 7(9), 122-133. http://dx.doi. org/10.7324/JAPS.2017.70917.

Vit, P., \& Santiago, B. (2008). Composición química de polen apícola fresco recolectado en el páramo de Misintá de los andes venezolanos. Archivos Latinoamericanos de Nutricion, 58(4), 411-415. PMid:19368304.
Vitali, D., Dragojević, I. V., \& Šebečić, B. (2009). Effects of incorporation of integral raw materials and dietary fibre on the selected nutritional and functional properties of biscuits. Food Chemistry, 114(4), 14621469. http://dx.doi.org/10.1016/j.foodchem.2008.11.032.

Yesiltas, B., Capanoglu, E., Firatligil-Durmus, E., Sunay, A. E., Samanci, T., \& Boyacioglu, D. (2014). Investigating the in-vitro bioaccessibility of propolis and pollen using a simulated gastrointestinal digestion system. Journal of Apicultural Research, 53(1), 101-108. http://dx.doi. org/10.3896/IBRA.1.53.1.10.

Žiaková, A., Brandšteterová, E., \& Blahová, E. (2003). Matrix solidphase dispersion for the liquid chromatographic determination of phenolic acids in Melissa officinalis. Journal of Chromatography. A, 983(1-2), 271-275. http://dx.doi.org/10.1016/S0021-9673(02)017120. PMid:12568390. 